\title{
Low vitamin $D$ and risk of bacterial pneumonias: Mendelian randomisation studies in two population- based cohorts
}

\author{
Yunus Çolak (ㄷ) 1,2 Børge G Nordestgaard, ${ }^{1,2,3}$ Shoaib Afzal (1) 1,2
}

\begin{abstract}
- Additional material is published online only. To view, please visit the journal online (http://dx.doi.org/10.1136/ thoraxjnl-2020-215288)

'Department of Clinical Biochemistry and the Copenhagen General Population Study, Herlev and Gentofte Hospital, Copenhagen University Hospital, Herlev, Denmark 2Faculty of Health and Medical Sciences, University of Copenhagen, Copenhagen, Denmark

${ }^{3}$ The Copenhagen City Heart Study, Frederiksberg Hospital, Copenhagen University Hospital, Copenhagen, Denmark
\end{abstract}

\section{Correspondence to}

Dr Shoaib Afzal, Department of Clinical Biochemistry and the Copenhagen General Population Study, Herlev and Gentofte Hospital, Copenhagen University Hospital, Herlev, Denmark 2730; shoaib.afzal@regionh.dk

Received 13 May 2020 Revised 10 September 2020 Accepted 8 October 2020

Published Online First

27 October 2020

\section{Linked}

- http://dx.doi.org/10.1136/ thoraxjnl-2020-216375

\section{Check for updates}

(C) Author(s) (or their employer(s)) 2021. No commercial re-use. See rights and permissions. Published by BMJ.

To cite: Çolak $Y$ Nordestgaard BG, Afzal S.

Thorax 2021;76:468-478.

\section{ABSTRACT \\ Background Vitamin D may regulate the innate} immune system, and randomised controlled trials suggest a beneficial effect of vitamin $D$ supplementation against acute respiratory tract infections. By using a Mendelian randomisation approach, we tested the hypothesis that low 25-hydroxyvitamin D is associated with increased risk of bacterial pneumonia in observational and genetic analyses.

Methods We genotyped 116335 randomly chosen white Danes aged 20 to 100 from the Copenhagen City Heart Study and Copenhagen General Population Study for plasma 25-hydroxyvitamin D decreasing genetic variants around CYP2R1 (rs117913124, rs12794714 and rs10741657), DHCR7 (rs7944926 and rs11234027), GEMIN2 (rs2277458) and HAL (rs3819817). Information on plasma 25-hydroxyvitamin D was available on 35833 individuals. Individuals were followed from 1981 through 2018 for hospital diagnoses of bacterial pneumonias.

Results During up to 38 years follow-up, we observed 6342 bacterial pneumonias in observational analyses and 13916 in genetic analyses. In observational analyses, multivariable adjusted HR for bacterial pneumonias was $1.27(95 \% \mathrm{Cl}: 1.16$ to 1.40$)$ for individuals with 25-hydroxyvitamin $\mathrm{D}<25 \mathrm{nmol} / \mathrm{L}$ compared with those with $\geq 25 \mathrm{nmol} / \mathrm{L}$. In genetic analyses, the OR for bacterial pneumonia per $10 \mathrm{nmol} / \mathrm{L}$ lower plasma 25-hydroxyvitamin D was 1.12 (95\% Cl: 1.02 to 1.23$)$ in Wald's ratio, 1.12 (95\% Cl: 1.04 to 1.20$)$ in inversevariance weighted, 1.63 (95\% Cl: 0.96 to 2.78$)$ in MR-Egger and 1.15 (95\% Cl: 1.05 to 1.26$)$ in weighted median instrumental variable analysis. This association was strongest for genetic variants around CYP2R1. There was no observational or genetic evidence to support that 25-hydroxyvitamin D is associated with risk of urinary tract infections, skin infections, sepsis or gastroenteritis, which were used as negative control outcomes.

Conclusions Low vitamin D is associated observationally and genetically with increased risk of bacterial pneumonias.

\section{INTRODUCTION}

The proposed role of vitamin D in human health has been extended well beyond calcium and bone homoeostasis to now include regulation of the innate immune system. ${ }^{12}$ Accordingly, observational studies have found that low plasma 25-hydroxyvitamin D concentrations are associated with increased risk of respiratory tract infections in adults, children and infants. ${ }^{3-9}$ Nevertheless,

\section{Key messages}

What is the key question?

- This study was designed to answer whether low plasma vitamin $D$ is a causal factor in bacterial pneumonias by using a Mendelian randomisation approach.

What is the bottom line?

- By using a Mendelian randomisation approach in two population-based cohorts with $>110000$ individuals and up to 38 years of follow-up, low plasma vitamin $\mathrm{D}$ is associated observationally and genetically with increased risk of bacterial pneumonias, but not with risk of urinary tract infections, skin infections, sepsis or gastroenteritis.

Why read on?

- This is the first study that investigates the causal association of vitamin D with bacterial pneumonias alone using genetic evidence.

randomised controlled trials investigating effect of vitamin D supplementation against respiratory tract infections have found conflicting results. ${ }^{10-23}$ However, a systematic review and meta-analysis comprising 11321 participants and including all randomised controlled trials with vitamin $\mathrm{D}$ supplementation of any duration against acute respiratory tract infections showed a beneficial effect. ${ }^{24}$ To our knowledge, no study has attempted to investigate the causal association of vitamin D with bacterial pneumonias alone using genetic evidence.

A Mendelian randomisation analysis takes advantage of natural randomisation and uses genetic variants as proxies of modifiable exposures. ${ }^{25}$ Since alleles are randomly distributed at conception, genetic variants should not be associated with potential confounders, and since genes are present at birth, genetic analyses are not susceptible to reverse causation. Thus, causal inferences can be made using such a study design akin to randomised controlled trials. Indeed, genetic variants have been identified that specifically lead to lifelong lower plasma 25-hydroxyvitamin D concentrations, ${ }^{26-29}$ providing an ideal framework to assess low 25-hydroxyvitamin D as a causal risk factor for bacterial pneumonias. In such a Mendelian randomisation framework, instruments or genetic variants known to be associated 
with plasma 25-hydroxyvitamin D are tested for their association with bacterial pneumonia. From this, it may be possible to show that 25 -hydroxyvitamin $\mathrm{D}$ is causal of bacterial pneumonia under a number of key assumptions. The assumptions are that the genetic variants are associated with 25 -hydroxyvitamin $\mathrm{D}$, are only associated with bacterial pneumonia through 25 -hydroxyvitamin D and are independent of confounders of the association of plasma 25-hydroxyvitamin $\mathrm{D}$ with bacterial pneumonia.

By using a Mendelian randomisation approach, we tested the hypothesis that low 25-hydroxyvitamin D is associated with increased risk of bacterial pneumonia in observational and genetic analyses. For this purpose, we used two populationbased cohorts with $>110000$ individuals and up to 38 years of follow-up.

\section{METHODS}

\section{Study populations}

The Copenhagen City Heart Study is a population-based prospective cohort study initiated in 1976 to 1978 , with follow-up examinations in 1981 to 1983,1991 to 1994 and 2001 to $2003 .{ }^{30}$ All individuals in Denmark are assigned a unique identification number at birth or immigration (Central Person Registration number) and recorded in the national Danish Civil Registration System. Individuals aged 20 to 100 were randomly selected and invited from the national Danish Civil Registration System to reflect the white adult general population of Danish descent (an individual is registered as of Danish descent in the national Danish Civil Registration System if the person and both parents are born in Denmark and have Danish citizenship). At study entry, all individuals completed a questionnaire, underwent a physical examination and gave blood for biochemical and genetic analyses (1991 to 1994 and 2001 to 2003 examinations only). In observational analyses, we included 10272 individuals with plasma 25 -hydroxyvitamin D measurements from the 1981 to 1983 examination. In genetic analyses, we included 9536 individuals from the 1991 to 1994 and/or 2001 to 2003 examinations with information on all genotypes. Of these, 5597 had information on both plasma 25-hydroxyvitamin D measurements and all genotypes.

The Copenhagen General Population Study is a populationbased prospective cohort study initiated in 2003 with ongoing enrolment, recruited and examined as the Copenhagen City Heart Study but from different parts of Copenhagen. ${ }^{30}$ In observational analyses, we included 25561 individuals, with plasma 25-hydroxyvitamin D measurements. In genetic analyses, we included 106799 individuals with information on all genotypes. Of these, 25410 had information on both plasma 25-hydroxyvitamin D measurements and all genotypes.

No individual appeared in more than one study. For the Copenhagen City Heart Study, the response-rate was $72 \%$ in the 1976 to 1978 examination, $63 \%$ in the 1981 to 1983 examination, $61 \%$ in the 1991 to 1994 examination and 50\% in the 2001 to 2003 examination. Non-responders versus responders in the 1976 to 1978 examination were more often men (52\% vs $46 \%$ ) and slightly older (median age: 55 vs 54 years). Similar differences could be observed between non-responders and responders for the subsequent examinations: $53 \%$ vs $45 \%$ and 61 vs 58 years in the 1981 to 1983 examination, 45\% vs 44\% and 63 vs 61 years in the 1991 to 1994 examination and 43\% vs $43 \%$ and 67 vs 62 years in the 2001 to 2003 examination, respectively. For the Copenhagen General Population Study recruiting in 2003 to 2015, response rate was $43 \%$. Non-responders versus responders were more often men (48\% vs 45\%) and slightly younger (median age: 56 vs 58 ).

\section{Plasma 25-hydroxyvitamin D measurements}

Measurements of plasma 25-hydroxyvitamin D using the DiaSorin Liaison 25(OH)vitamin D Total Assay, a direct competitive chemiluminescence immunoassay, were conducted blind to information on outcome and genotypes. ${ }^{30}$ For the Copenhagen City Heart Study, plasma samples were collected in 1981 to $1983(\mathrm{n}=10272)$ and stored at $-20^{\circ} \mathrm{C}$ for approximately 30 years before measurement. For the Copenhagen General Population Study, plasma samples were either collected in 2004 to $2005(\mathrm{n}=12506)$ and stored at $-80^{\circ} \mathrm{C}$ for approximately 5 years before measurement or in 2009 to $2011(n=13$ 055) for measurement on fresh samples. Assay precision was tested daily, while assay accuracy was tested monthly using an external quality control programme (Vitamin D External Quality Assessment Scheme (DEQAS)). The inter-assay coefficient of variance was $10 \%$ for low concentration control $(\sim 40 \mathrm{nmol} / \mathrm{L})$ and $8 \%$ for high concentration control $(\sim 135 \mathrm{nmol} / \mathrm{L})$.

\section{Genotypes}

Genotyping using TaqMan assays was conducted blind to information on outcome and plasma 25-hydroxyvitamin D concentrations. ${ }^{30}$ Specific genotypes for plasma 25 -hydroxyvitamin D concentration were selected as those having the strongest and largest association in genome-wide association studies, ${ }^{26} 29$ we chose genetic variants around CYP2R1 (rs12794714 and rs10741657) and DHCR7 (rs7944926 and rs11234027), as they are expected to influence biologically active plasma 25 -hydroxyvitamin D concentrations via either synthesis of previtamin D from 7-dehydrocholesterol in the skin or conversion of vitamin D to 25 -hydroxyvitamin $\mathrm{D}$ in the liver. We on purpose did not genotype for variants in the vitamin $\mathrm{D}$ binding protein, as such variants will influence the amount of 25 -hydroxyvitamin $\mathrm{D}$ transported in plasma rather than differences in biologically active plasma 25-hydroxyvitamin D. Recently, three additional genotypes for plasma 25-hydroxyvitamin D concentration were identified in genome-wide association studies; a rare genetic variant located around CYP2R1 (rs117913124) and two frequent genetic variants around GEMIN2 (rs2277458) and HAL (rs3819817), ${ }^{27} 28$ which we also genotyped. Call rates were $>99 \%$ after two reruns.

\section{Bacterial pneumonias}

Bacterial pneumonias (International Classification of Diseases (ICD)-8: 481xx to 486xx and ICD-10: A481, J13 to J16, J170, J18) were defined as acute emergency department visits and hospital admissions with the mentioned primary diagnosis. Information was obtained from the national Danish Patient Registry, which records all public and private hospital contacts in Denmark, recorded from baseline until 13 December 2018. Diagnoses recorded in the registry are made by medical doctors according to national law. Denmark used the ICD-8 until 1 January 1994 and proceeded directly to ICD-10 after this date. As negative control outcomes, we included urinary tract infections, skin infections, sepsis and gastroenteritis. ${ }^{31}$

By using the unique Central Person Registration number provided to everyone in Denmark at birth or immigration and linking it with the national Danish Patient Registry, no person was lost to follow-up, and individuals who emigrated were censored at the date of emigration $(n=137$ in observational analyses and $n=538$ in genetic analyses). 


\section{Potential confounders}

Body mass index (BMI) was calculated as measured weight divided by measured height squared $\left(\mathrm{kg} / \mathrm{m}^{2}\right)$. Smoking status was defined as never, former or current smoking. Cumulated tobacco consumption was calculated in pack-years based on information on age at smoking initiation and cessation (or for current smokers until age at baseline examination), duration of tobacco consumption and amount of consumed tobacco in form of number of daily consumed cigarettes, cheroots and cigars and grams of weekly consumed pipe tobacco: a pack-year was defined as 20 cigarettes or equivalent smoked daily for a year. Alcohol consumption was self-reported in units per week ( 1 unit $=12$ g). Estimated glomerular filtration rate (eGFR) was calculated from serum creatinine measured using standard hospital assays. Chronic disease at baseline included diabetes mellitus, ischaemic heart disease, chronic obstructive pulmonary disease (COPD) and cancer. Diabetes at baseline was based on self-report, nonfasting plasma glucose $>11 \mathrm{mmol} / \mathrm{L}$, use of antidiabetic medication and/or previous inpatient/outpatient hospital contacts due to diabetes (ICD-8: 249 to 250 and ICD-10: E10 to E14), obtained from the national Danish Patient Registry. Ischaemic heart disease (ICD-8: 410 to 414 and ICD-10: I20 to I25) and COPD (ICD-8: 491 to 492 and ICD-10: J41 to J44) at baseline were based on inpatient/outpatient hospital contacts, obtained from the national Danish Patient Registry. Cancer at baseline, excluding non-melanoma skin cancer cases, was obtained from the national Danish Cancer Registry.

\section{Statistical analyses}

Deviation from Hardy-Weinberg equilibrium was investigated using the $\chi^{2}$-tests; deviation may suggest genotyping or population sampling errors, but we observed none. Associations with potential confounders were investigated using linear and logistic regression models, as appropriate. Associations between the genotypes and plasma 25-hydroxyvitamin D were investigated using linear regression models; we evaluated the strength of the genotypes as instruments by examining the F-statistic and $\mathrm{R}^{2}$ : a high $\mathrm{F}$ and/or $\mathrm{R}^{2}$ indicates sufficient strength to ensure statistical reliability of the risk estimates. ${ }^{32}$ Kaplan-Meier estimator was used to determine cumulative prevalence of bacterial pneumonias with log-rank test. Cox proportional hazard models were used to determine risk of bacterial pneumonias. Multiple failure-time analysis was carried out to investigate risk of recurrent events using the method proposed by Andersen and Gill. ${ }^{33}$ Wald's test was used to assess interaction on risk of bacterial pneumonias. We used age as the underlying timescale (=age adjusted) with left truncation (= delayed entry) at study examination. Observational analyses were adjusted for age, sex, seasonal variation of 25 -hydroxyvitamin D (by adjusting for month and year of blood sampling), birth year, cohort, BMI, smoking status, cumulative tobacco consumption, alcohol consumption, eGFR and chronic disease at baseline (diabetes, ischaemic heart disease, COPD and cancer). Genetic analyses were adjusted for age, sex, birth year and cohort, as genetic analyses generally are not prone to confounding. In order to estimate the causal effect of plasma 25 -hydroxyvitamin D on bacterial pneumonia, we used instrumental variable analysis with individual level data, accounting for partial overlap and methods designed to be robust to some violations of the assumptions for Mendelian randomisation analyses, that is, inverse-variance weighted, MR-Egger and weighted median estimators. ${ }^{34} 35$ All instrumental variable analyses were carried out using single events, that is, first event, as methodology for investigating recurrent events has not been developed yet. The causal effect was estimated by including genetic variants that were not prone to weak instrument bias (sufficiently high F-statistic and $\mathrm{R}^{2}$ ) in accordance with previous recommendations. ${ }^{32}{ }^{36}$ However, instrumental variable analysis was repeated by also including all genetic variants. In a sensitivity analysis, we investigated the associations in the UK Biobank through GeneAtlas. ${ }^{37}$ However, UK Biobank had a different outcome definition (influenza and all pneumonia combined) from the one used in the present study, as bacterial pneumonia alone was not available. In another sensitivity analysis, we combined genetic variants around CYP2R1 to create unweighted and weighted genetic risk score, since most of the association seemed to be driven through these genotypes; only internal weighted risk scores could be calculated as genome-wide association studies did not report results necessary to calculate externally weighted risk score. ${ }^{26-29}$ Since information on potential confounders was 99.9\% complete, we performed multivariate imputation using chained equations to fill out the missing values in the observational analyses; however, results were similar without the use of imputation. We used Stata/SE 13.1 for Windows.

\section{RESULTS}

We had 35833 individuals in observational analyses and 116335 in genetic analyses (figure 1 and table 1). Plasma 25-hydroxyvitamin D concentrations were associated with all potential confounders (online supplemental table S1). In contrast, the genotypes for 25 -hydroxyvitamin D were not associated with any potential confounders (online supplemental table S2) but as expected associated with plasma 25-hydroxyvitamin $\mathrm{D}$ concentrations in a dose-dependent manner (online supplemental figure S1), illustrating that such genotypes can be used as largely unconfounded instruments to assess the association of genetically determined low 25-hydroxyvitamin D with risk of bacterial pneumonias by using a Mendelian randomisation approach. Among the genotypes, GEMIN2 rs2277458 and HAL rs3819817 had F-statistic $<10$ and $\mathrm{R}^{2}<0.1 \%$, suggesting that these genotypes may be weak instruments (online supplemental figure S1).

In the Copenhagen City Heart Study, median follow-up was 21 years (up to 38 years) for the observational analyses and 18 years (up to 27 years) for the genetic analyses. In the Copenhagen General Population Study, corresponding values were 8.8 years (up to 14 years) and 9.3 years (up to 15 years), respectively. During follow-up, we observed 6342 bacterial pneumonias in the observational analyses and 13916 in the genetic analyses.

\section{Observational analyses}

Individuals with 25 -hydroxyvitamin $\mathrm{D}<25 \mathrm{nmol} / \mathrm{L}$ had an increased risk of bacterial pneumonias compared with those with 25 -hydroxyvitamin $\mathrm{D} \geq 25 \mathrm{nmol} / \mathrm{L}$ in the Copenhagen City Heart Study and Copenhagen General Population Study, both in combined and separate analyses (figure 2). Compared with individuals with 25 -hydroxyvitamin $\geq 25 \mathrm{nmol} / \mathrm{L}$, the multivariable adjusted HR for bacterial pneumonias was 1.27 (95\% CI: 1.16 to 1.40 ) in individuals with 25 -hydroxyvitamin $\mathrm{D}<25 \mathrm{nmol} / \mathrm{L}$. This risk estimate was largely similar in subgroup analyses without evidence of interaction, including in the two studies separately (figure 3).

Compared with individuals with 25 -hydroxyvitamin $\geq 50 \mathrm{nmol} / \mathrm{L}$, multivariable adjusted HRs for bacterial pneumonias were 0.95 (95\% CI: 0.88 to 1.103 ) in those with 25 to $49.9 \mathrm{nmol} / \mathrm{L}, 1.19$ (95\% CI: 1.06 to 1.32 ) in those with 12.5 to $24.9 \mathrm{nmol} / \mathrm{L}$ and 1.51 (95\% CI: 1.25 
Observational analyses flowchart

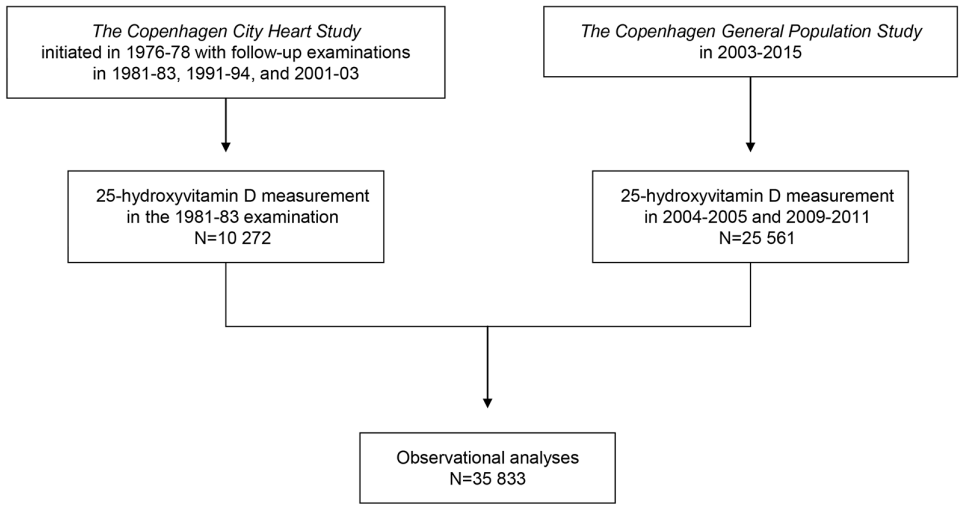

Genetic analyses flowchart

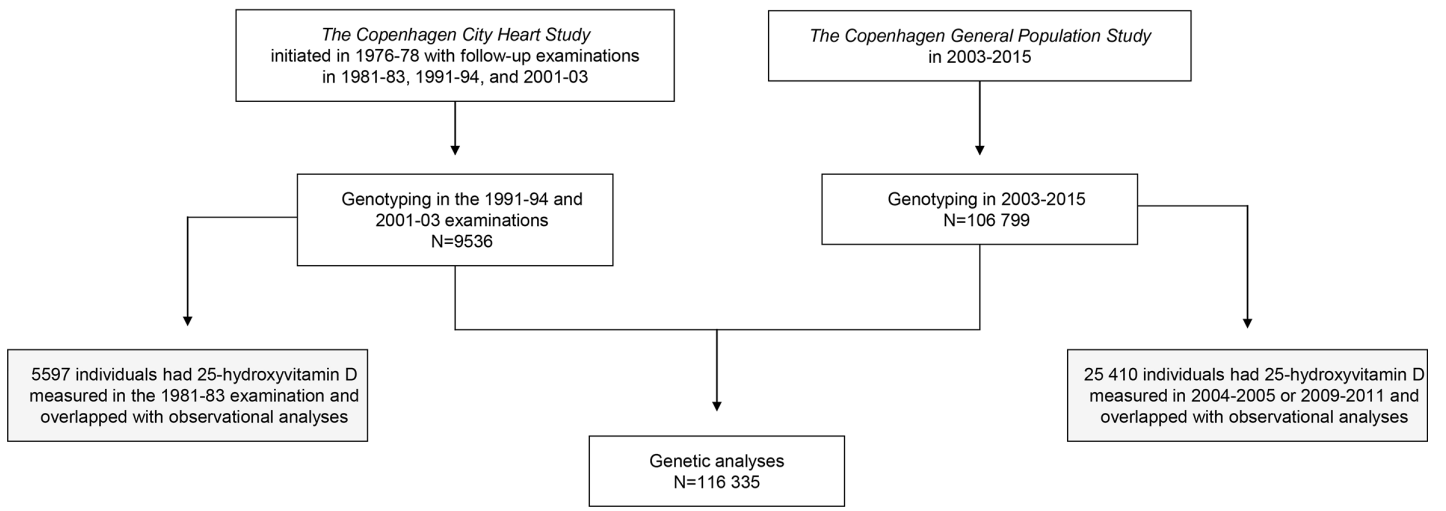

Figure 1 Flowchart depicting samples used in observational and genetic analyses.

to 1.81$)$ in those with $<12.5 \mathrm{nmol} / \mathrm{L} 25$-hydroxyvitamin D (figure 4). No clear associations were observed between 25-hydroxyvitamin D concentrations and risk of urinary tract infections, skin infections, sepsis or gastroenteritis (all p values for trend $\geq 0.05$ ); however, 95\% CIs for HRs did not overlap with 1.0 in individuals with plasma 25-hydroxyvitamin $\mathrm{D}<12.5 \mathrm{nmol} / \mathrm{L}$ for skin infections and $12.5-24.9 \mathrm{nmol} / \mathrm{L}$ for sepsis.

Table 1 Potential confounders in observational and genetic analyses in individuals in the general population

\begin{tabular}{|c|c|c|c|c|}
\hline & $\begin{array}{l}\text { Individuals in } \\
\text { observational analyses } \\
(\mathrm{n}=35 \text { 833) }\end{array}$ & $\begin{array}{l}\text { Association with } \\
25 \text {-hydroxyvitamin D } \\
\text { concentration, P value* }\end{array}$ & $\begin{array}{l}\text { Individuals in genetic } \\
\text { analyses } \\
(\mathrm{n}=116335)\end{array}$ & $\begin{array}{l}\text { Association with } \\
\text { genetic risk score, } \\
\text { P value* }^{*}\end{array}$ \\
\hline Age - years & 58.1 (48.8 to 66.6$)$ & $4 \times 10^{-13}$ & $58.3(48.1$ to 67.7$)$ & 0.23 \\
\hline Men - no. (\%) & $16076(45)$ & $5 \times 10^{-28}$ & $52339(45)$ & 0.26 \\
\hline $\mathrm{BMI}-\mathrm{kg} / \mathrm{m}^{2}$ & 25.4 (23.0 to 28.2$)$ & $2 \times 10^{-116}$ & 25.5 (23.1 to 28.4$)$ & 0.91 \\
\hline Active smokers - no. (\%) & $11230(31)$ & $5 \times 10^{-286}$ & $22787(20)$ & 0.16 \\
\hline Cumulative tobacco consumption - pack-years $\dagger$ & $20.0(9.0$ to 35.0$)$ & $2 \times 10^{-98}$ & $16.5(6.3$ to 31.0$)$ & 0.96 \\
\hline Alcohol - units/day & $1.0(0.3$ to 2.0$)$ & 0.002 & $1.1(0.4$ to 2.1$)$ & 0.72 \\
\hline $\mathrm{eGFR}-\mathrm{mL} / \mathrm{min}$ & 77 (65 to 89$)$ & $9 \times 10^{-43}$ & 80 (69 to 91$)$ & 0.68 \\
\hline \multicolumn{5}{|l|}{ Chronic disease at baseline } \\
\hline Diabetes - no. (\%) & $1507(4)$ & $2 \times 10^{-10}$ & $4945(4)$ & 0.30 \\
\hline Ischaemic heart disease & $1925(5)$ & 0.001 & $6616(6)$ & 0.79 \\
\hline COPD - no. (\%) & $725(2)$ & 0.02 & $2405(2)$ & 0.39 \\
\hline Cancer - no. (\%) & 2091 (6) & $2 \times 10^{-6}$ & 7798 (7) & 0.27 \\
\hline
\end{tabular}

Data presented as median $\left(25^{\text {th }}\right.$ and $75^{\text {th }}$ percentiles), or $\mathrm{n}(\%)$. Based on the Copenhagen City Heart Study and the Copenhagen General Population Study.

*Calculated using linear or logistic regression, as appropriate.

tOnly calculated for former and current smokers.

BMI, body mass index; COPD, chronic obstructive pulmonary disease; eGFR, estimated glemarular filtration rate. 
The Copenhagen City Heart Study and Copenhagen General Population Study

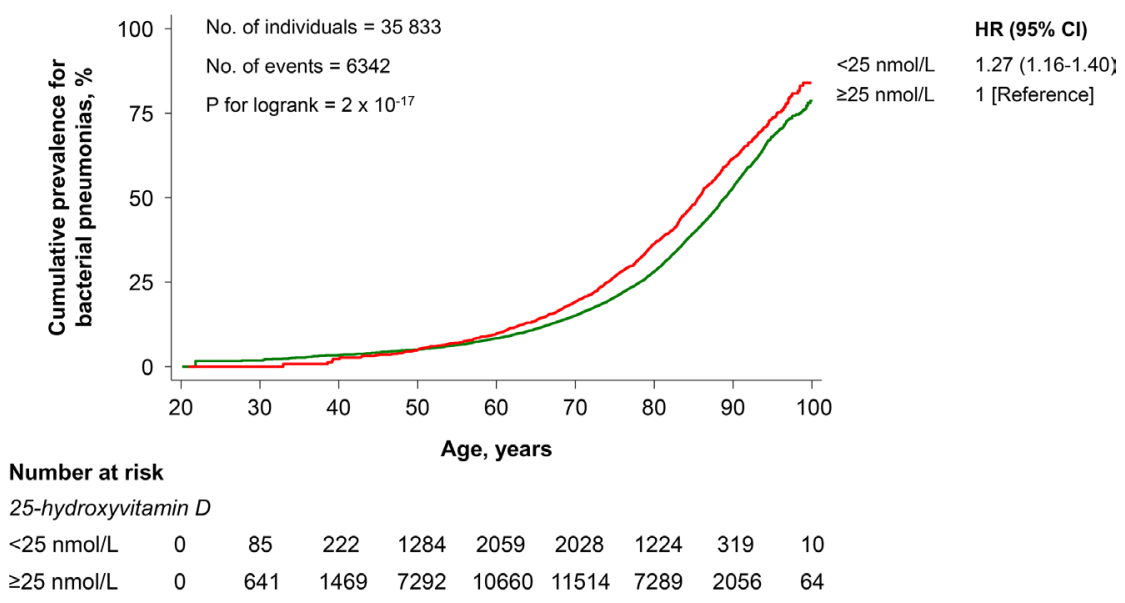

The Copenhagen City Heart Study

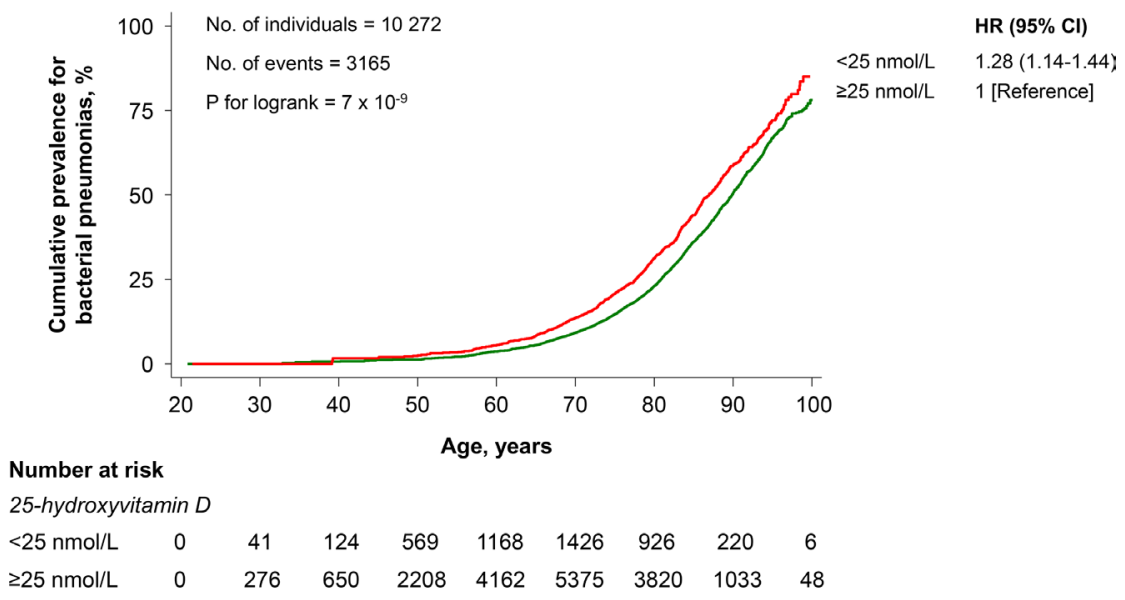

The Copenhagen General Population Study

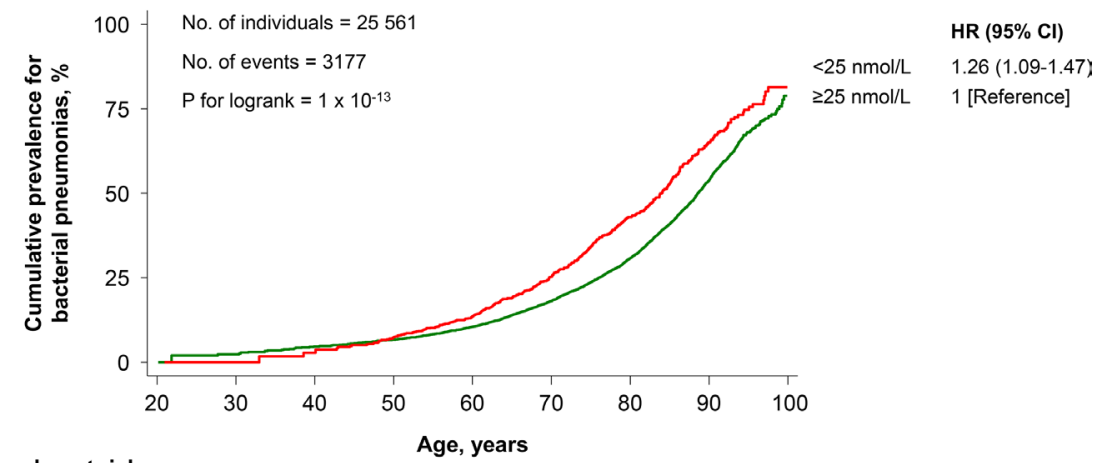

Number at risk

25-hydroxyvitamin $D$

$\begin{array}{llllllllll}<25 \mathrm{nmol} / \mathrm{L} & 0 & 44 & 98 & 715 & 891 & 602 & 298 & 99 & 4\end{array}$

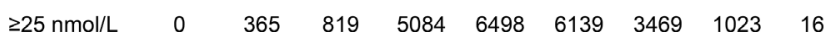

Figure 2 Cumulative prevalence and risk of bacterial pneumonias according to plasma 25-hydroxyvitamin D concentration in individuals in the general population. Cumulative prevalence from Kaplan-Meier estimator, and risk estimates obtained from Cox regression models with multiple failure-time analysis according to Andersen and Gill adjusted for age (as timescale), sex, seasonal variation of 25-hydroxyvitamin D, birth year, cohort, body mass index, smoking status, cumulative tobacco consumption, alcohol consumption, estimated glomerular filtration rate, chronic disease at baseline (diabetes, ischaemic heart disease, chronic obstructive pulmonary disease and cancer). 


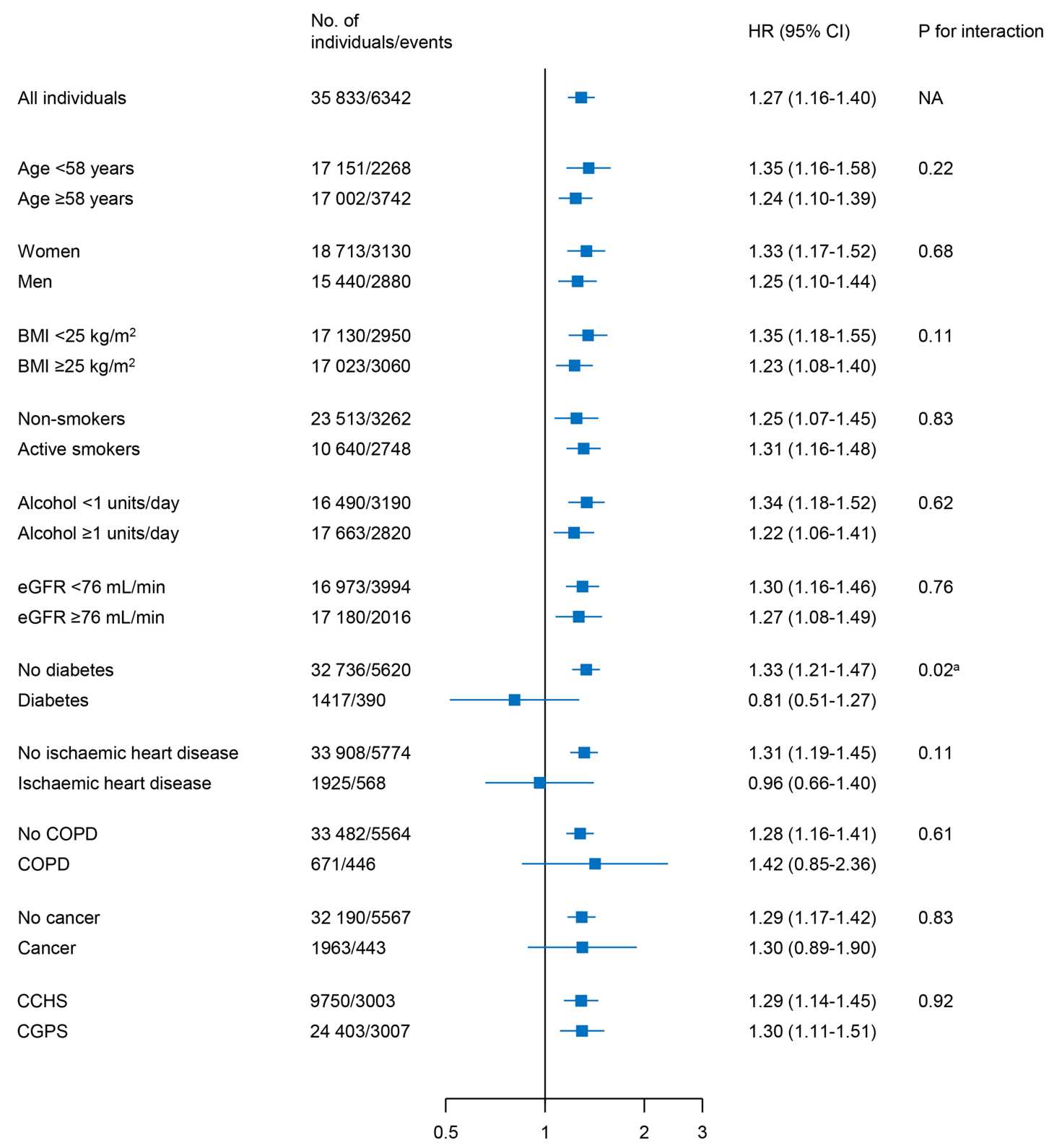

$\mathrm{HR}(95 \% \mathrm{Cl})$ for bacterial pneumonias for $<25 \mathrm{nmol} / \mathrm{L}$ versus $\geq 25 \mathrm{nmol} / \mathrm{L}$ 25-hydroxyvitamin $D$

Figure 3 Risk of bacterial pneumonias in different subgroups according to plasma 25-hydroxyvitamin D concentration in individuals in the general population. Risk estimates obtained from Cox regression models with multiple failure-time analysis according to Andersen and Gill adjusted for age (as timescale), sex, seasonal variation of 25-hydroxyvitamin D, birth year, cohort, body mass index (BMI), smoking status, cumulative tobacco consumption, alcohol consumption, estimated glomerular filtration rate (eGFR), chronic disease at baseline (diabetes, ischaemic heart disease, chronic obstructive pulmonary disease (COPD) and and cancer), as appropriate. P for interaction obtained from Wald's test. Number of individuals vary slightly in the subgroup analyses due to availability of data. Chosen cut-off values for age, BMI, alcohol and eGFR represent median values. Based on the Copenhagen City Heart Study (CCHS) and Copenhagen General Population Study (CGPS) if not otherwise stated. Risk estimates for the CCHS and CGPS vary slightly from figure 1 , as complete case analysis has been used here.

\section{Genetic analyses}

Each 25-hydroxyvitamin D decreasing allele in CYP2R1 rs12794714 and CYP2R1 rs10741657 was associated with higher risk of bacterial pneumonias but not of other infections (figure 5). Other genotypes were not associated individually with higher risk of bacterial pneumonias or other infections; however, individuals with genetic variation in GEMIN2 rs2277458 had a reduced risk of sepsis and gastroenteritis.

In instrumental variable analysis, lower plasma 25-hydroxyvitamin D concentration was associated with higher risk of bacterial pneumonia (figures 6 and 7). ORs for bacterial pneumonia per $10 \mathrm{nmol} / \mathrm{L}$ lower plasma 25 -hydroxyvitamin D were respectively 1.12 (95\% CI: 1.02 to 1.23 ) in Wald's ratio, 1.12 (95\% CI: 1.04 to 1.20 ) in inverse-variance weighted, 1.63 (95\% CI: 0.96 to 2.78 ) in MR-Egger and 1.15 (95\% CI: 1.05 to 1.26$)$ in weighted median instrumental variable analysis (figure 7, upper panel). Results were attenuated but similar when also including the rare genetic variant, that is, CYP2R1 rs117913124, and the genetic variants prone to weak instrument bias, that is, GEMIN2 rs2277458 and HAL rs3819817 (figure 7, 
No. of

individuals/events

Bacterial pneumonias

25-hydroxyvitamin D

$\geq 50 \mathrm{nmol} / \mathrm{L}$

$25-49.9 \mathrm{nmol} / \mathrm{L}$

$12.5-24.9 \mathrm{nmo} / \mathrm{L}$

$<12.5 \mathrm{nmol} / \mathrm{L}$

Urinary tract infections

25-hydroxyvitamin D

$\geq 50 \mathrm{nmol} / \mathrm{L}$

$25-49.9 \mathrm{nmol} / \mathrm{L}$

$12.5-24.9 \mathrm{nmol} / \mathrm{L}$

$<12.5 \mathrm{nmol} / \mathrm{L}$

\section{Skin infections}

25-hydroxyvitamin D

$\geq 50 \mathrm{nmol} / \mathrm{L}$

$25-49.9 \mathrm{nmol} / \mathrm{L}$

$12.5-24.9 \mathrm{nmol} / \mathrm{L}$

$<12.5 \mathrm{nmol} / \mathrm{L}$

\section{Sepsis}

25-hydroxyvitamin D

$\geq 50 \mathrm{nmol} / \mathrm{L}$

$25-49.9 \mathrm{nmol} / \mathrm{L}$

$12.5-24.9 \mathrm{nmol} / \mathrm{L}$

$<12.5 \mathrm{nmol} / \mathrm{L}$

\section{Gastroenteritis}

25-hydroxyvitamin D

$\geq 50 \mathrm{nmol} / \mathrm{L}$

$25-49.9 \mathrm{nmol} / \mathrm{L}$

$12.5-24.9 \mathrm{nmol} / \mathrm{L}$

$<12.5 \mathrm{nmol} / \mathrm{L}$

$888 / 246$

$4081 / 321$

$888 / 93$

$17762 / 1199$

13 102/1011

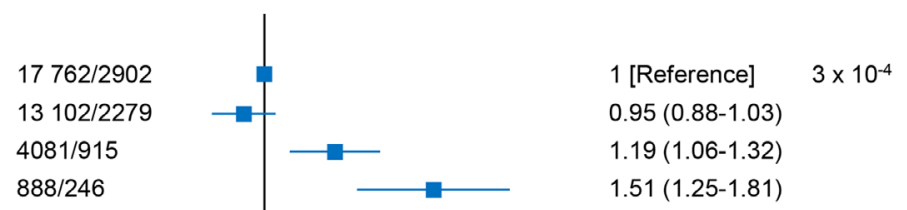

$1.51(1.25-1.81)$

1 [Reference] $\quad 0.08$

1.03 (0.94-1.13)

$1.12(0.96-1.30)$

$1.21(0.94-1.56)$

1 [Reference] 0.20

$1.00(0.90-1.12)$

$1.00(0.86-1.17)$

$1.42(1.08-1.87)$

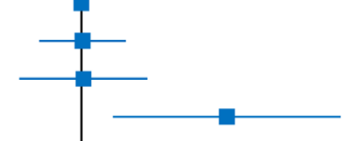

1 [Reference] 0.11

0.98 (0.86-1.12)

$1.24(1.01-1.52)$

$1.17(0.81-1.68)$

1 [Reference] $\quad 0.27$

$1.09(0.94-1.27)$

$1.06(0.84-1.34)$

$1.23(0.82-1.87)$

Figure 4 Risk of bacterial pneumonias and other infections according to plasma 25-hydroxyvitamin D concentration in individuals in the general population. Risk estimates obtained from Cox regression models with multiple failure-time analysis according to Andersen and Gill adjusted for age (as timescale), sex, seasonal variation of 25-hydroxyvitamin D, birth year, cohort, body mass index, smoking status, cumulative tobacco consumption, alcohol consumption, estimated glomerular filtration rate, chronic disease at baseline (diabetes, ischaemic heart disease, chronic obstructive pulmonary disease and cancer). Based on the Copenhagen City Heart Study and Copenhagen General Population Study.

lower panel). However, these latter analyses may be biassed as outliers affect inverse-variance weighted and MR-Egger estimates, and weak instruments can also bias estimates in general, though usually towards the observational estimate.

\section{Sensitivity analyses}

None of the 25-hydroxyvitamin D decreasing genotypes were associated with risk of influenza and all pneumonia combined in the UK Biobank, and estimates were attenuated for the Copenhagen City Heart Study and Copenhagen General Population Study compared with bacterial pneumonia alone (online supplemental figure S2); however, the CYP2R1 rs12794714 genotype was associated with increased risk of influenza and all pneumonia in the Copenhagen City Heart Study and Copenhagen General Population Study (latter also as a separate cohort). However, this outcomes definition is different from our main analyses as UK Biobank estimates could not be attained for bacterial pneumonia alone.

Since we suspected that the increased risk of bacterial pneumonia was probably driven through CYP2R1, we combined all available genetic variants around CYP2R1 (rs117913124, rs12794714 and rs10741657) in a genetic risk score. HR per 1,25-hydroxyvitamin D decreasing CYP2R1 risk score for bacterial pneumonias was 1.03 (95\% CI: 1.01 to 1.04 ) in an unweighted genetic risk score and 1.01 (95\% CI: 1.00 to 1.02 ) in a weighted genetic risk score (online supplemental figure S3). 
No. of

individuals/events

per allele

Bacterial pneumonias

CYP2R1 rs117913124

CYP2R1 rs12794714

CYP2R1 rs10741657

DHCR7 rs7944926

DHCR7 rs11234027

GEMIN2 rs2277458

HAL rs3819817

Urinary tract infections

CYP2R1 rs117913124

CYP2R1 rs12794714

CYP2R1 rs10741657

DHCR7 rs7944926

DHCR7 rs11234027

GEMIN2 rs2277458

HAL rs3819817

Skin infections

CYP2R1 rs117913124

CYP2R1 rs12794714

CYP2R1 rs 10741657

DHCR7 rs7944926

DHCR7 rs11234027

GEMIN2 rs2277458

HAL rs3819817

Sepsis

CYP2R1 rs117913124

CYP2R1 rs12794714

CYP2R1 rs10741657

DHCR7 rs7944926

DHCR7 rs11234027

GEMIN2 rs2277458

HAL rs 3819817

Gastroenteritis

CYP2R1 rs117913124

CYP2R1 rs12794714

CYP2R1 rs10741657

DHCR7 rs7944926

DHCR7 rs11234027

GEMIN2 rs2277458

HAL rs3819817

$\begin{array}{ll}117283 / 13722 & -8.5 \mathrm{nmol} / \mathrm{L}(-13 \%) \\ 117130 / 13718 & -2.8 \mathrm{nmol} / \mathrm{L}(-4.2 \%) \\ 117531 / 13759 & -2.5 \mathrm{nmol} / \mathrm{L}(-3.8 \%) \\ 117465 / 13743 & -2.0 \mathrm{nmol} / \mathrm{L}(-4.1 \%) \\ 117261 / 13700 & -2.1 \mathrm{nmol} / \mathrm{L}(-4.4 \%) \\ 117503 / 13752 & -1.3 \mathrm{nmol} / \mathrm{L}(-2.1 \%) \\ 117468 / 13743 & -0.9 \mathrm{nmol} / \mathrm{L}(-2.0 \%)\end{array}$

117 283/9687

117 130/9672

$117531 / 9719$

117 465/9706

117 261/9690

$117503 / 9714$

117 468/9711

117 130/7239

$117531 / 7258$

$117465 / 7249$

$117261 / 7231$

$117503 / 7260$

$117468 / 7252$

$117283 / 3948$

117 130/3933

$117531 / 3949$

$117465 / 3950$

$117261 / 3935$

$117503 / 3950$

$117468 / 3951$

117 283/2559

117 130/2561

$117531 / 2568$

117 465/2563

117 261/2555

$117503 / 2568$

117 468/2565
$-8.5 \mathrm{nmol} / \mathrm{L}(-13 \%)$

$-2.8 \mathrm{nmol} / \mathrm{L}(-4.2 \%)$

$-2.5 \mathrm{nmol} / \mathrm{L}(-3.8 \%)$

$-2.0 \mathrm{nmol} / \mathrm{L}(-4.1 \%)$

$-2.1 \mathrm{nmol} / \mathrm{L}(-4.4 \%)$

$-1.3 \mathrm{nmol} / \mathrm{L}(-2.1 \%)$

$-0.9 \mathrm{nmol} / \mathrm{L}(-2.0 \%)$

$-8.5 \mathrm{nmol} / \mathrm{L}(-13 \%)$

$-2.8 \mathrm{nmol} / \mathrm{L}(-4.2 \%)$

$-2.0 \mathrm{nmol} / \mathrm{L}(-4.1 \%)$

$-2.1 \mathrm{nmol} / \mathrm{L}(-4.4 \%)$

$-1.3 \mathrm{nmol} / \mathrm{L}(-2.1 \%)$

$-0.9 \mathrm{nmol} / \mathrm{L}(-2.0 \%)$

$-8.5 \mathrm{nmol} / \mathrm{L}(-13 \%)$

$-2.8 \mathrm{nmol} / \mathrm{L}(-4.2 \%)$

$-2.5 \mathrm{nmol} / \mathrm{L}(-3.8 \%)$

$-2.0 \mathrm{nmol} / \mathrm{L}(-4.1 \%)$

$-2.1 \mathrm{nmol} / \mathrm{L}(-4.4 \%)$

$-1.3 \mathrm{nmol} / \mathrm{L}(-2.1 \%)$

$-0.9 \mathrm{nmol} / \mathrm{L}(-2.0 \%)$

$-8.5 \mathrm{nmol} / \mathrm{L}(-13 \%)$

$-2.8 \mathrm{nmol} / \mathrm{L}(-4.2 \%)$

$-2.5 \mathrm{nmol} / \mathrm{L}(-3.8 \%)$

$-2.0 \mathrm{nmol} / \mathrm{L}(-4.1 \%)$

$-2.1 \mathrm{nmol} / \mathrm{L}(-4.4 \%)$

$-1.3 \mathrm{nmol} / \mathrm{L}(-2.1 \%)$

$-0.9 \mathrm{nmol} / \mathrm{L}(-2.0 \%)$
$-2.5 \mathrm{nmol} / \mathrm{L}(-3.8 \%)$

\section{vitamin D}

.

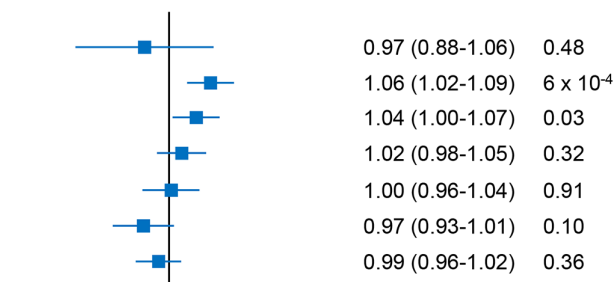

$1.00(0.89-1.11) \quad 0.95$ $1.00(0.96-1.03) \quad 0.87$

$1.01(0.97-1.04) \quad 0.77$

$0.99(0.95-1.02) \quad 0.46$

$0.98(0.93-1.02) \quad 0.30$

$0.99(0.94-1.04) \quad 0.74$

$1.02(0.98-1.05) \quad 0.37$

$1.01(0.91-1.13) \quad 0.80$

$0.98(0.94-1.01) \quad 0.22$

$1.00(0.96-1.03) \quad 0.82$

$1.03(0.99-1.07) \quad 0.16$

$1.02(0.97-1.06) \quad 0.51$

$1.01(0.95-1.06) \quad 0.85$

$0.98(0.94-1.02) \quad 0.25$

$1.00(0.86-1.16) \quad 0.97$

$1.00(0.95-1.05) \quad 0.93$

$1.02(0.97-1.08) \quad 0.37$

$1.00(0.94-1.05) \quad 0.88$

$1.00(0.94-1.07) \quad 0.99$

$0.91(0.85-0.97) \quad 0.01$

$1.04(0.99-1.09) \quad 0.15$

$0.96(0.80-1.15) \quad 0.68$

$1.02(0.96-1.08) \quad 0.54$

$1.06(1.00-1.13) \quad 0.07$

$0.99(0.93-1.06) \quad 0.74$

$0.97(0.90-1.04) \quad 0.41$

$0.92(0.85-0.99) \quad 0.04$

$1.02(0.96-1.08) \quad 0.49$

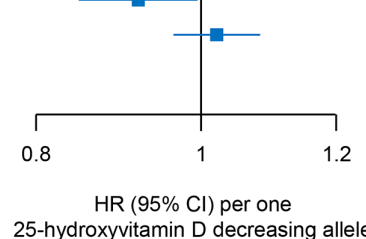

25-hydroxyvitamin D decreasing allele

Figure 5 Risk of bacterial pneumonias and other infections according to 25-hydroxyvitamin D decreasing genotypes in individuals in the general population. Risk estimates obtained from Cox regression models with multiple failure-time analysis according to Andersen and Gill adjusted for age (as timescale), sex, birth year and cohort. Based on the Copenhagen City Heart Study and Copenhagen General Population Study.

\section{DISCUSSION}

By using a Mendelian randomisation approach in two populationbased cohorts with $>110000$ individuals and up to 38 years of follow-up, we found that low plasma vitamin D is associated observationally and genetically with increased risk of bacterial pneumonias. There was no convincing evidence observationally or genetically that vitamin $\mathrm{D}$ is associated with risk of urinary tract infections, skin infections, sepsis or gastroenteritis.

Vitamin D seems to regulate the innate immune system, which is essential in the defence against bacterial pneumonias. $^{2}$ It has been shown that toll-like receptor (TLR)-2/1 ligand, T-cell-derived interferon-gamma (IFN- $\gamma$ ) and T-cellexpressed CD40 ligand induce upregulation of vitamin D receptor (VDR) and 25-hydroxyvitamin D-1- $\alpha$-hydroxylase (CYP27B1) (enzyme that converts inactive 25-hydroxyvitamin $\mathrm{D}$ to active 1,25-hydroxyvitamin D) through an autocrine pathway in human monocytes and macrophages. ${ }^{39-43}$ VDR and 1,25-hydroxyvitamin $\mathrm{D}$ then induce upregulation of cathelicidinrelated antimicrobial peptides, that is, LL-37 and hCAP-18, and human $\beta$-defensin 2, autophagy and phagosome maturation, which ultimately leads to intracellular killing of pathogens, as 


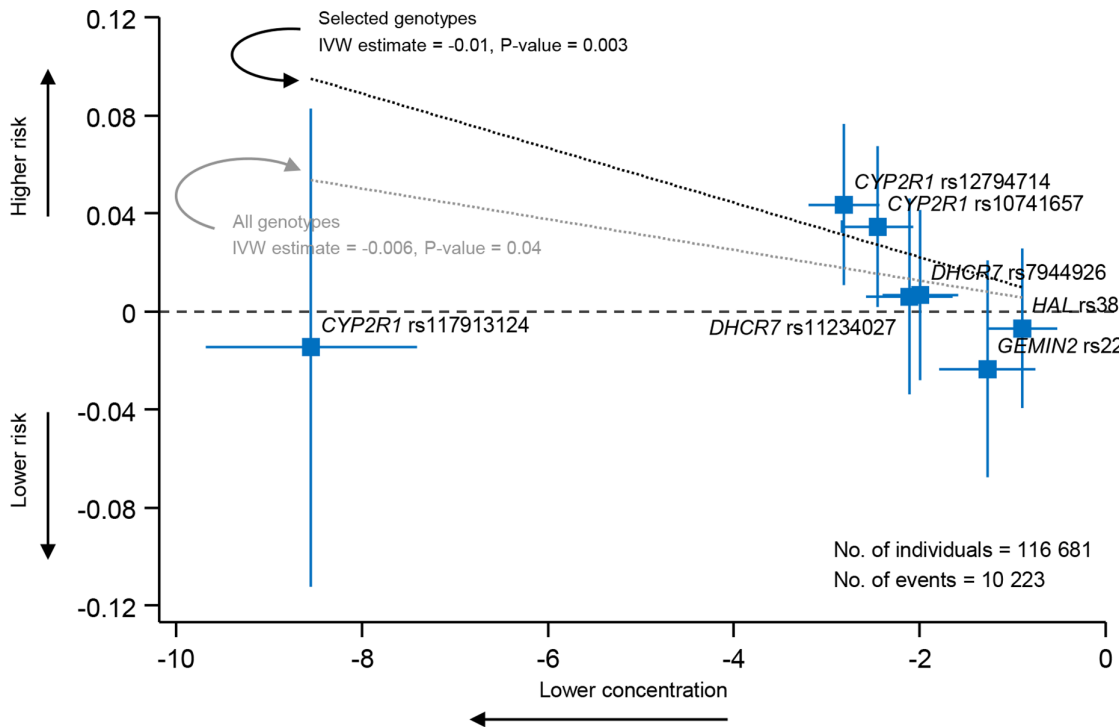

25-hydroxyvitamin D nmol/L (95\% Cl)

Figure 6 Beta coefficient for bacterial pneumonia and change in plasma 25-hydroxyvitamin D concentration D according to 25-hydroxyvitamin D decreasing genotypes in individuals in the general population. Beta coefficients for bacterial pneumonia obtained from logistic regression models adjusted for age, sex, birth year and cohort. Change in plasma 25-hydroxyvitamin D concentration obtained from multiple linear regression models adjusted for age, sex, seasonal variation of 25-hydroxyvitamin D, birth year and cohort. Dashed black (selected genotypes) and grey (all genotypes) lines obtained from inverse-variance weighted (IVW) instrumental variable analysis. Selected genotypes included CYP2R1 rs12794714, CYP2R1 rs10741657, DHCR7 rs7944926 and DHCR7 rs11234027. Based on the Copenhagen City Heart Study and Copenhagen General Population Study.

shown for Mycobacterium tuberculosis, Pseudomonas aeruginosa and Bordetella bronchiseptica. ${ }^{39-44}$

Interestingly, genetic variants around CYP2R1 but not around DHCR7, GEMIN2 or HAL were associated individually with increased risk of bacterial pneumonias. A potential explanation may be that CYP2R1 is more directly involved in the formation of 1,25-hydroxyvitamin D compared with the other genes. Also, while the role of DHCR7 in vitamin D metabolism is well-established (conversion of 7-dehydrocholesterol to cholesterol), the role of GEMIN2 and HAL is unknown. This may also explain the low F-statistic and $\mathrm{R}^{2}$ of genetic variants around GEMIN2 and HAL compared with genetic variants around CYP2R1 and DHCR7. Also, we cannot exclude that variants in these genes may only indirectly affect 25 -hydroxyvitamin D through pathways affecting both exposure an outcome separately (pleiotropy). Further, we cannot exclude the possibility
No. of

individuals/events

IV analysis with selected genotypes

Wald's ratio

Inverse-variance weighted

MR-Egger

Weighted median

IV analysis with all genotypes

Wald's ratio

Inverse-variance weighted

MR-Egger

Weighted median

$116681 / 10223$

$116681 / 10223$

$116681 / 10223$
$116681 / 10223$

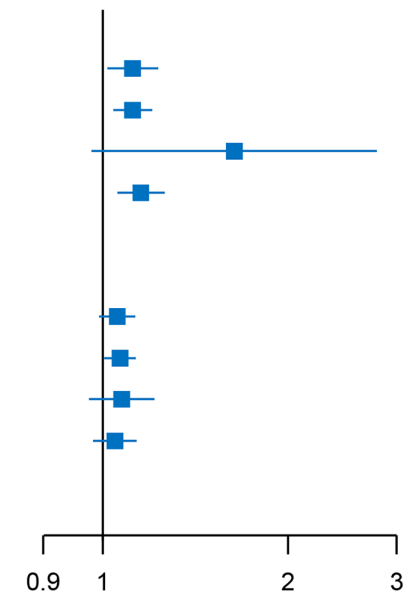

OR $(95 \% \mathrm{Cl})$ for bacterial pneumonia

per $10 \mathrm{nmol} / \mathrm{L}$ lower plasma 25-hydroxyvitamin D

Figure 7 Risk of bacterial pneumonia according to 25-hydroxyvitamin D concentration using instrumental variable analysis in individuals in the general population. Risk estimates for bacterial pneumonia obtained from logistic regression models adjusted for age, sex, birth year and cohort. Change in plasma 25-hydroxyvitamin D concentration obtained from multiple linear regression models adjusted for age, sex, seasonal variation of 25-hydroxyvitamin D, birth year and cohort. Selected genotypes included CYP2R1 rs12794714, CYP2R1 rs10741657, DHCR7 rs7944926 and DHCR7 rs11234027. Based on the Copenhagen City Heart Study and Copenhagen General Population Study. IV, instrumental variable. 
that vitamin D may not be involved in the defence against bacterial pneumonias, and that other mechanisms involving CYP2R1 may be at play. Finally, the association of genetic variants around CYP2R1 with risk of bacterial pneumonias could represent a chance finding and therefore needs independent confirmation.

We were unable to replicate our findings in the UK Biobank. A potential explanation may be that a different outcome was used, that is, influenza and all pneumonia combined instead of bacterial pneumonia alone. Although we found evidence of an association with influenza and all pneumonia for the 25-hydroxyvitamin D decreasing CYP2R1 rs12794714 genotype, we believe that this association was probably driven by bacterial pneumonia. Another potential explanation may be that events were identified differently in the UK Biobank and the validity of these endpoints in UK biobank is unknown. Information on events in the present study was obtained from the national Danish Patient Registry, which records all public and private hospital contacts in Denmark, where diagnoses are made by medical doctors according to national law. In addition, events were only defined as acute emergency department visits and hospital admissions based on the primary diagnosis to increase the validity, ${ }^{45}$ and to secure that we only captured the most serious cases admitted to hospital in the Copenhagen cohorts.

Previous observational studies have found an association of low plasma 25-hydroxyvitamin D concentrations with higher risk of respiratory tract infections. ${ }^{3-9}$ Although results from randomised controlled trials testing the effect of vitamin D supplementation against respiratory tract infections have been conflicting, ${ }^{10-23}$ the largest systematic review and meta-analysis to date has pointed at a beneficial effect. ${ }^{24}$ In the present study, we show that low vitamin $\mathrm{D}$ is associated with increased risk of bacterial pneumonias in observational analyses and in part in genetic analyses. The fact that there was no evidence observationally or genetically that vitamin D is associated with risk of other organ specific infections suggests that the association could be specific for respiratory infections such as bacterial pneumonias. Future randomised controlled trials should therefore be designed to study the effect of vitamin D supplementation against bacterial pneumonias.

Strengths of the present study include two large-scale independent cohorts from the general population with randomly selected individuals with a long follow-up time, no losses to follow-up and a substantial number of bacterial pneumonias. Furthermore, we used physician encoded diagnoses with high validity. ${ }^{46}$

Potential limitations in Mendelian randomisation analyses include population stratification bias, genetic pleiotropy and linkage disequilibrium. However, as we had an ethnically homogeneous white Danish population, the complicating effects of population stratification were likely avoided, and as genotype distributions did not appear to differ according to the HardyWeinberg equilibrium, genotyping and population sampling errors were also likely avoided.

Another potential limitation is that we did not have information on family relationships, which could bias our results. However, since we have sampled randomly, we believe that this bias will be of minor importance.

As mentioned above, we cannot exclude that our positive genetic observation could not represent a chance finding. However, it is reassuring that all different instrumental variable analyses that were used to estimate the causal effect, each with its belonging strengths and weaknesses,${ }^{34}$ gave similar results, suggesting that the results are robust.

In conclusion, low vitamin $\mathrm{D}$ is associated observationally and genetically with increased risk of bacterial pneumonias.
Collaborators NA.

Contributors $Y C$ and SA had full access to all the data in the study and take responsibility for the integrity of the data and the accuracy of the data analyses. YÇ, BGN and SA contributed to the study concept and design. YÇ, BGN and SA collected, analysed or interpreted the data. YÇ wrote the draft manuscript and did the statistical analyses. YÇ, BGN and SA revised the manuscript for important intellectual content. BGN obtained funding. BGN provided administrative, technical or material support. BGN and SA supervised the study.

Funding The Lundbeck Foundation. The funding source had no role in the design and conduct of the study; collection, management, analysis or interpretation of the data; preparation, review or approval of the manuscript; or decision to submit the manuscript for publication.

Competing interests $Y C$ reports personal fees from Boehringer Ingelheim, AstraZeneca, Sanofi Genzyme outside the submitted work. BGN and SA have nothing to disclose.

Patient consent for publication Not required.

Ethics approval Both studies were approved by Danish ethical committees and conducted according to the Declaration of Helsinki (approval numbers: KF-V-1002039/91 and H-KF-01-144/01). All individuals provided written informed consent.

Provenance and peer review Not commissioned; externally peer reviewed.

Data availability statement Data are available upon reasonable request. Additional data regarding technical details, statistical code and derivative data are available from the corresponding author. Data access for further analyses is possible through direct collaborative agreement or through locally managed access arranged through the study's principal investigator.

\section{ORCID iDs}

Yunus Çolak http://orcid.org/0000-0003-2045-829X

Shoaib Afzal http://orcid.org/0000-0002-1442-4694

\section{REFERENCES}

1 Holick MF. Vitamin D deficiency. N Eng/ J Med 2007;357:266-81.

2 Kroner JdeC, Sommer A, Fabri M. Vitamin D every day to keep the infection away? Nutrients 2015;7:4170-88.

3 Feng $\mathrm{H}$, Xun $\mathrm{P}$, Pike $\mathrm{K}$, et al. In utero exposure to 25-hydroxyvitamin D and risk of childhood asthma, wheeze, and respiratory tract infections: A meta-analysis of birth cohort studies. J Allergy Clin Immunol 2017;139:1508-17.

4 Ginde AA, Mansbach JM, Camargo CA. Association between serum 25-hydroxyvitamin D level and upper respiratory tract infection in the third National health and nutrition examination survey. Arch Intern Med 2009:169:384-90.

5 Jovanovich AJ, Ginde AA, Holmen J, et al. Vitamin D level and risk of communityacquired pneumonia and sepsis. Nutrients 2014;6:2196-205.

6 Łuczyńska A, Logan C, Nieters A, et al. Cord blood 25(OH)D levels and the subsequent risk of lower respiratory tract infections in early childhood: the Ulm birth cohort. Eur J Epidemiol 2014;29:585-94.

7 Laaksi I, Ruohola JP, Tuohimaa P, et al. An association of serum vitamin D concentrations. Am J Clin Nutr 2007;86:714-7.

8 Pacheco-González RM, García-Marcos L, Morales E. Prenatal vitamin D status and respiratory and allergic outcomes in childhood: a meta-analysis of observational studies. Pediatr Allergy Immunol 2018;29:243-53.

9 Robertsen S, Grimnes G, Melbye H. Association between serum 25-hydroxyvitamin D concentration and symptoms of respiratory tract infection in a Norwegian population: the Tromsø study. Public Health Nutr 2014;17:780-6.

10 Aglipay M, Birken CS, Parkin PC, et al. Effect of high-dose vs standard-dose Wintertime vitamin $\mathrm{D}$ supplementation on viral upper respiratory tract infections in young healthy children. JAMA 2017;318:245-54.

11 Bergman P, Lindh AU, Björkhem-Bergman L, et al. Vitamin D and respiratory tract infections: a systematic review and meta-analysis of randomized controlled trials. PLoS One 2013;8:e65835

12 Chandy DD, Kare J, Singh SN, et al. Effect of vitamin D supplementation, directly or via breast milk for term infants, on serum 25 hydroxyvitamin D and related biochemistry, and propensity to infection: a randomised placebo-controlled trial. $\mathrm{Br} J$ Nutr 2016;116:52-8.

13 Ginde AA, Blatchford P, Breese K, et al. High-Dose monthly vitamin D for prevention of acute respiratory infection in older long-term care residents: a randomized clinical trial. J Am Geriatr Soc 2017;65:496-503.

14 Goodall EC, Granados AC, Luinstra K, et al. Vitamin D3 and gargling for the prevention of upper respiratory tract infections: a randomized controlled trial. BMC Infect Dis 2014;14:273.

15 Grant CC, Kaur S, Waymouth E, et al. Reduced primary care respiratory infection visits following pregnancy and infancy vitamin D supplementation: a randomised controlled trial. Acta Paediatr 2015;104:396-404. 
16 Li-Ng M, Aloia JF, Pollack S, et al. A randomized controlled trial of vitamin D3 supplementation for the prevention of symptomatic upper respiratory tract infections. Epidemiol Infect 2009;137:1396-404.

17 Mao S, Huang S. Vitamin D supplementation and risk of respiratory tract infections: a meta-analysis of randomized controlled trials. Scand J Infect Dis 2013;45:696-702.

18 Martineau AR, Hanifa Y, Witt KD, et al. Double-Blind randomised controlled trial of vitamin D3 supplementation for the prevention of acute respiratory infection in older adults and their carers (ViDiFlu). Thorax 2015;70:953-60.

19 Martineau AR, James WY, Hooper RL, et al. Vitamin D3 supplementation in patients with chronic obstructive pulmonary disease (ViDiCO): a multicentre, double-blind, randomised controlled trial. Lancet Respir Med 2015;3:120-30.

20 Martineau AR, MacLaughlin BD, Hooper RL, et al. Double-Blind randomised placebocontrolled trial of bolus-dose vitamin D3 supplementation in adults with asthma (ViDiAs). Thorax 2015;70:451-7.

21 Murdoch DR, Slow S, Chambers ST, et al. Effect of vitamin D3 supplementation on upper respiratory tract infections in healthy adults: the VIDARIS randomized controlled trial. JAMA 2012;308:1333-9.

22 Rees JR, Hendricks K, Barry EL, et al. Vitamin D3 supplementation and upper respiratory tract infections in a randomized, controlled trial. Clin Infect Dis 2013;57:1384-92

23 Vuichard Gysin D, Dao D, Gysin CM, et al. Effect of vitamin D3 supplementation on respiratory tract infections in healthy individuals: a systematic review and metaanalysis of randomized controlled trials. PLoS One 2016;11:e0162996.

24 Martineau AR, Jolliffe DA, Hooper RL, et al. Vitamin D supplementation to prevent acute respiratory tract infections: systematic review and meta-analysis of individual participant data. BMJ 2017:356:i6583.

25 Smith GD, Ebrahim S. 'Mendelian randomization': can genetic epidemiology contribute to understanding environmental determinants of disease? Int J Epidemiol 2003;32:1-22.

26 Ahn J, Yu K, Stolzenberg-Solomon R, et al. Genome-Wide association study of circulating vitamin D levels. Hum Mol Genet 2010;19:2739-45.

27 Manousaki D, Dudding T, Haworth S, et al. Low-Frequency synonymous coding variation in CYP2R1 has large effects on vitamin D levels and risk of multiple sclerosis. Am J Hum Genet 2017;101:227-38.

28 Jiang X, O'Reilly PF, Aschard H, et al. Genome-Wide association study in 79,366 European-ancestry individuals informs the genetic architecture of 25-hydroxyvitamin D levels. Nat Commun 2018;9:260.

29 Wang TJ, Zhang F, Richards JB, et al. Common genetic determinants of vitamin D insufficiency: a genome-wide association study. Lancet 2010;376:180-8.

30 Afzal S, Brøndum-Jacobsen P, Bojesen SE, et al. Vitamin D concentration, obesity, and risk of diabetes: a Mendelian randomisation study. Lancet Diabetes Endocrinol 2014;2:298-306.
31 Helby J, Nordestgaard BG, Benfield T, et al. Asthma, other atopic conditions and risk of infections in 105519 general population never and ever smokers. J Intern Med 2017;282:254-67.

32 Lawlor DA, Harbord RM, Sterne JAC, et al. Mendelian randomization: using genes as instruments for making causal inferences in epidemiology. Stat Med 2008;27:1133-63.

33 Andersen PK, Gill RD. Cox's regression model for counting processes: a large sample study. Ann Stat 1982;10:1100-20.

34 Slob EAW, Burgess S. A comparison of robust Mendelian randomization methods using summary data. Genet Epidemiol 2020;44:313-29.

35 Cosetta M, Fabiola Del Greco M, van der Plaat DA, et al. The use of two-sample methods for Mendelian randomization analyses on single large datasets. bioRxiv 2020

36 Swerdlow DI, Kuchenbaecker KB, Shah S, et al. Selecting instruments for Mendelian randomization in the wake of genome-wide association studies. Int J Epidemiol 2016:45:1600-16.

37 Canela-Xandri 0, Rawlik K, Tenesa A. An atlas of genetic associations in UK Biobank. Nat Genet 2018:50:1593-9.

38 Gene Atlas. Roslin Institute and MRC human genetics unit. University of Edinburgh. Available: http://geneatlas.roslin.ed.ac.uk/search/?traits=187\&variants= rs117913124+rs12794714+rs10741657+rs7944926+rs11234027+rs2277458+ rs3819817 [Accessed 21 Aug 2020].

39 Fabri M, Stenger S, Shin D-M, et al. Vitamin D is required for IFN-gamma-mediated antimicrobial activity of human macrophages. Sci Trans/ Med 2011;3:104ra102.

40 Klug-Micu GM, Stenger S, Sommer A, et al. Cd40 ligand and interferon- $\gamma$ induce an antimicrobial response against Mycobacterium tuberculosis in human monocytes. Immunology 2013;139:121-8.

41 Yuk J-M, Shin D-M, Lee H-M, et al. Vitamin D3 induces autophagy in human monocytes/macrophages via cathelicidin. Cell Host Microbe 2009;6:231-43.

42 Liu PT, Schenk M, Walker VP, et al. Convergence of IL-1beta and VDR activation pathways in human TLR2/1-induced antimicrobial responses 2009;4:e5810.

43 Liu PT, Stenger S, Li H, et al. Toll-Like receptor triggering of a vitamin D-mediated human antimicrobial response. Science 2006;311:1770-3.

44 Yim S, Dhawan P, Ragunath C, et al. Induction of cathelicidin in normal and CF bronchial epithelial cells by 1,25-dihydroxyvitamin D(3). J Cyst Fibros 2007;6:403-10.

45 Benfield T, Jensen JS, Nordestgaard BG. Influence of diabetes and hyperglycaemia on infectious disease hospitalisation and outcome. Diabetologia 2007;50:549-54.

46 Schmidt M, Schmidt SAJ, Sandegaard JL, et al. The Danish national patient registry: a review of content, data quality, and research potential. Clin Epidemiol 2015:7:449-90. 